\title{
Embarazo en pacientes con lesión medular
}

\author{
Verónica Serrano de la Cruz D. ${ }^{1}$; Alicia Martínez V. ${ }^{1}$; María José Núnez V. ${ }^{1}$; \\ Laura Giménez R. ${ }^{1}$; Vicente Diago A. ${ }^{1}$; Alfredo Perales M. ${ }^{1}$ \\ ${ }^{1}$ Área de la Salud de la Mujer, Servicio de Ginecología y Obstetricia, Hospital Universitario y Politécnico La Fe. Valencia, \\ España.
}

\section{RESUMEN}

Antecedentes: Las gestaciones en pacientes con daño medular representan un reto para la obstetricia. Las complicaciones más frecuentes son las infecciones de las vías respiratorias y de las vías urinarias, y la más grave la hiperreflexia autonómica. El parto vaginal es posible en lesiones por debajo de T5, pero por encima de ese nivel, la imposibilidad de percibir las contracciones, de identificar el trabajo de parto y realizar pujos lleva a la necesidad de una cesárea programada. Objetivo: Exponer nuestra experiencia y revisar el manejo más adecuado de la embarazada parapléjica. Métodos: Estudio retrospectivo en el periodo 2003-2014. Hubo 10 pacientes con 24 gestaciones: 6 abortos espontáneos, un 1 aborto inducido, quedando para el análisis 17 gestaciones (1 embarazo gemelar). La evaluación es independiente a la etiología o localización de la lesión. Se analiza las complicaciones perinatales y maternas. Resultados: La edad media fue 33 años. La edad gestacional media al parto fue 37 semanas: 12 de término (70,59\%), 4 de pretérmino (23,53\%) y una desconocida (5,88\%). Hubo 10 cesáreas $(58,82 \%)$ y 7 partos vaginales (41,18\%). Peso medio neonatal de $2940 \mathrm{~g}$, ninguno con retraso de crecimiento intrauterino. Conclusiones: Nuestra experiencia muestra una alta tasa de prematuridad y de cesáreas, pero con buenos resultados maternos y perinatales, que hace permisible que estas pacientes puedan cumplir su deseo genésico, superando su discapacidad.

\section{PALABRAS CLAVE: Paraplejia, embarazo, pronóstico materno-perinatal}

\section{SUMMARY}

Background: The pregnancies in patients with spinal cord injury represent a challenge for obstetrics. The most common complications are infections of the respiratory and urinary tract, and the most severe is the autonomic hyperreflexia. Vaginal delivery is possible in lesions below T5, but above that level the inability to perceive contractions, to identify labor and perform straining leads to the need for a scheduled cesarean. Objective: To describe our experience and review the most appropriate management of paraplegic pregnant. Methods: Retrospective study in the period 2003-2014. There were 10 patients with 24 pregnancies: 6 spontaneous abortions, 1 induced abortion, leaving 17 for analysis pregnancies (one twin pregnancy). The evaluation is independent of the etiology or location of the lesion. Perinatal and maternal complications were analyzed. Results: The mean age was 33 years. The mean gestational age at delivery was 37 weeks: 12 to term $(70.59 \%), 4$ preterm deliveries $(23.53 \%)$ and one unknow. There were 10 cesarean sections $(58.82 \%)$ and 7 vaginal deliveries (41.18\%). Average birth weight of $2940 \mathrm{~g}$, none with intrauterine growth restriction. Conclusions: Our experience shows a high rate of prematurity and cesarean section, but with good maternal and perinatal outcomes, which makes it permissible for these patients can fulfill their procreative desire, overcoming his disability.

KEY WORD: Paraplegia, pregnancy, maternal perinatal outcome 


\section{INTRODUCCIÓN}

Las gestaciones en pacientes con daño medular se están convirtiendo en un reto cada vez más frecuentes en el trabajo de los obstetras. Pueden producirse a cualquier nivel medular, desde la zona cervical a la sacra, siendo la localización de la lesión lo que determina qué sistemas se verán afectados.

Como resultado, las pacientes pueden ser tetrapléjicas, cuando se pierde la actividad motora y sensitiva en el tronco, brazos y piernas, o parapléjicas, cuando la pérdida se limita al tronco y los miembros inferiores $(1,2)$. La deambulación se ve afectada y esa incapacidad para movilizarse puede afectar la capacidad respiratoria, el flujo cardiovascular, la continencia urinaria, la movilidad de la vejiga, el manejo del peso, la consistencia ósea y la salud mental, lo que implica compromiso de la calidad de vida de la paciente.

En EEUU más de 11.000 casos de lesión medular ocurren cada año, afectando la mitad de ellos a población entre 15 y 25 años, alcanzando a 3000 mujeres en edad fértil (2). En el caso de pacientes jóvenes, la causa más frecuente son los accidentes del tráfico. Actualmente este tipo de lesiones, gracias al avance en el tratamiento de la lesión aguda, la rehabilitación y los esfuerzos por integrar a estas mujeres, ha permitido que un mayor número estén completamente adaptadas a las necesidades sociales y de la vida diaria, de modo que se plantean su deseo de ser madres.

En los últimos 20 años se ha observado un aumento del número de pacientes afectadas de lesión medular que requieren atención obstétrica. Pese a su discapacidad, las mujeres con este tipo de lesiones tienen la función reproductora y sexual totalmente conservada, en contraste con lo que sucede en los varones con este tipo de afectación (3). El embarazo en este tipo de mujeres es posible como en cualquier otra, pero se trata de embarazos de alto riesgo y requiere un abordaje multidisciplinar (4). En estas circunstancias es elemental una planificación adecuada para decidir el momento de la búsqueda de una gestación.

La frecuencia de embarazos en estas pacientes sigue siendo baja con respecto a la población sana, y a consecuencia de la falta de experiencia, las conductas obstétricas no son uniformes. El objetivo de nuestro estudio es exponer nuestra experiencia en embarazadas parapléjicas y revisar el manejo materno-perinatal.

\section{PACIENTES Y MÉTODOS}

Estudio retrospectivo de las gestaciones en pacientes con lesiones medulares que fueron seguidas en nuestro hospital entre los años 2003 y 2014. Se recabaron 10 pacientes con daño medular con 24 gestaciones, analizamos 17 gestaciones (se excluyen 6 abortos espontáneos y una interrupción voluntaria del embarazo). Fueron evaluadas, independientemente de su etiología o localización de la lesión. Hemos recogido la causa y nivel lesional, la edad de la paciente en el embarazo, el año de la gestación, la historia obstétrica, la edad gestacional al parto, las complicaciones durante la gestación, parto y puerperio, el tipo de finalización, la analgesia en el parto y los resultados perinatales.

\section{RESULTADOS}

En el período analizado se han manejado 24 gestaciones en 10 pacientes. La edad materna media fue 33 años (rango: 23-41 años). Una finalizó en interrupción voluntaria del embarazo, 6 fueron abortos espontáneos en gestaciones iniciales, 12 gestaciones finalizaron a término (una de ellas gemelar bicorial biamniótica), 4 pretérmino (en uno de los casos desconocemos la edad gestacional de finalización).

Las complicaciones maternas observadas fueron 3 infecciones del tracto urinario (2 a repetición), 2 casos con alto riesgo de trombosis y necesidad de profilaxis antitrombótica, 1 caso de hipotiroidismo gestacional y 1 de diabetes gestacional. Otra de las gestaciones sufrió amenaza de parto pretérmino, rotura prematura de membranas pretérmino y requirió finalizar el embarazo a las 36 semanas por fiebre materna. Finalmente, el caso más complicado aconteció en una gestante parapléjica secundaria de un mielomeningocele, con insuficiencia renal crónica que se agravó por preeclampsia y obligó a finalizar el embarazo a las 33 semanas mediante cesárea.

En 10 pacientes se interrumpió el embarazo por cesárea (8 programadas y 2 de urgencia). Siete mediante parto vaginal (6 partos espontáneos y un vacuum extractor). La analgesia durante el parto fue en 8 casos anestesia general $(47,06 \%)$, en 2 casos raquídea $(11,76 \%)$, en 2 pacientes epidural $(11,76 \%)$ y en 5 local $(29,41 \%)$. La edad gestacional media al parto fue de 37 semanas (rango: 33-41 semanas): 12 a término (70,59\%), 4 de pretérmino $(23,53 \%)$ y en uno de los casos desconocemos la edad gestacional a la que se finalizó el embarazo $(5,88 \%)$. Se obtuvieron 18 recién nacidos con peso medio de 2940 gramos (rango: 2030-3650 g), ninguno pequeño para edad gestacional ni afecto de retraso de crecimiento intrauterino.

En la Tabla I se presentan los datos sobre la etiología y nivel de la lesión medular.

\section{DISCUSIÓN}

En la actualidad, no existe acuerdo basado en ensayos clínicos en las recomendaciones para el cuidado óptimo de estas pacientes durante el embarazo. Hasta el momento el conocimiento se basa en series de casos y estudios casos control (1). Una revisión sobre el manejo de las complicaciones urológicas no pudo extraer conclusiones útiles para la práctica clínica por el mismo motivo la literatura es limitada (5). 


\section{Tabla I}

\section{ETIOLOGÍA Y NIVEL DE LA LESIÓN EN 10 EMBARAZADAS PARAPLÉJICAS}

\begin{tabular}{l}
\hline Etiología de la lesión \\
\hline accidentes de tráfico (50\%) \\
1 accidente laboral (10\%) \\
1 accidente deportivo (10\%) \\
2 tumores medulares (20\%) \\
1 iatrógenica (10\%) \\
Nivel de la lesión \\
Paciente 1. T12-L5 \\
Paciente 2. T2 \\
Paciente 3. T4 \\
Paciente 4. T7 izquierda, T10 derecha \\
Paciente 5. T5 \\
Paciente 6. T4 izquierda, T8 derecha \\
Paciente 7. Desconocido \\
Paciente 8. T12 \\
Paciente 9. T10 \\
Paciente 10. Desconocido
\end{tabular}

Desde que la paciente plantea el deseo de ser madre, hay que insistir en la necesidad de planificar el embarazo adecuadamente, porque se trata de una gestación de alto riesgo, y se requiere valorar los tratamientos y necesidades de la paciente previa a la gestación.

Al igual que en la población general, hay que dar una serie de recomendaciones generales y optimizar en medida de lo posible la situación física y mental de la paciente previa a la gestación. Salvo que haya indicación por antecedentes personales y/o familiares, no es necesario hacer un estudio genético, porque las lesiones medulares maternas no incrementan el riesgo de alteraciones de ese tipo, luego el proceder será idéntico al de la población general.

Ansiedad y depresión son dos complicaciones frecuentes en este tipo de mujeres tanto en el embarazo como en el año posterior a la maternidad. No hay consenso en cuanto al manejo de estas complicaciones, pero es evidente que la utilización de antidepresivos está contraindicada por teratogenia fetal y por interaccionar con otros fármacos pudiendo aumentar la espasticidad. La primera línea de tratamiento en estos casos son la acupuntura y la terapia cognitivo conductual (1).

La valoración y modificación de los tratamientos habituales de la paciente previa a la gestación es obligatoria, la medicación para el control del dolor, la espasticidad, la regulación de la tensión arterial, la profilaxis de la infección de la orina y la depresión, no son adecuados durante el embarazo, por lo cual hay que sustituirlos o retirarlos de forma anticipada. Si la paciente ha tomado tratamientos antiepilépticos es necesario suplir previo a la gestación y durante la misma la carencia de ácido fólico con $4 \mathrm{mg}$ diarios, para intentar evitar los defectos del tubo neural. Es útil administrar suplementos de hierro, porque la existencia de anemia bien ferropénica, megaloblástica o por trastornos crónicos es habitual.

Debido a la tendencia habitual de estas pacientes al estreñimiento, y al posible agravamiento de la situación con el embarazo y el tratamiento con hierro es conveniente tomar algunas medidas. La evacuación intestinal con supositorios o con ayuda de estimulación digital, ejercicios de evacuación o determinadas pautas dietéticas pude ser insuficiente para mantener el ritmo intestinal habitual previo. Para conseguirlo, puede ser necesario añadir o aumentar la dosis de ablandadores de heces suaves, agentes aumentadores de volumen y laxantes. El manejo del estreñimiento es importante, no sólo por las molestias que puede causar a la paciente, sino porque la incapacidad de evacuar o la impactación de heces puede desencadenar hiperreflexia autónoma. Ocasionalmente se requiere manejo multidisciplinar de la paciente para conseguir controlar esta situación (1).

Las complicaciones más frecuentemente asociadas a este tipo de embarazos son la anemia (deficiencia de hierro, deficiencia de folato, anemia por enfermedad crónica, insuficiencia renal crónica), infecciones del tracto urinario, úlceras por decúbito, contracturas musculares, estreñimiento, trombosis venosa profunda (TVP), compromiso respiratorio, parto de pretérmino y síndrome de disrreflexia autonómica. En nuestra casuística tuvimos ITUs de repetición, riesgo de TVP, insuficiencia renal y aumento de la prematuridad.

Debido a la tendencia a la espasticidad y las contracturas, los ejercicios de refuerzo de la musculatura en los miembros superiores puede estar recomendados en la mayoría de los pacientes, pero es conveniente valorar de forma personalizada cada caso para pautar el tipo de ejercicios que puede ser útil en función de la localización y el grado de lesión medular (3).

Las úlceras de decúbito se asocian al mantenimiento de una postura de forma mantenida, y se localizan habitualmente en muslos, espalda y nalgas. El embarazo aumenta el riesgo de úlceras por el aumento de peso, la sequedad de la piel y el cambio de puntos de presión en la pelvis. Debe comprobarse la integridad de la piel de la gestante diariamente en su domicilio y al final de cada valoración por la matrona o el tocólogo. Existen una serie de medidas higiénico dietéticas que pueden disminuir la tendencia a la producción de úlceras (1). 
Preservar la función renal de estas pacientes, incluso fuera del embarazo, es un reto importante pues la elevada presión intracavitaria vesical a consecuencia de la baja compliance de la vejiga y la hiperactividad del detrusor llevan a hidronefrosis y este es la principal causa de deterioro renal en estas pacientes. Los principales problemas urinario de estas gestantes son las infecciones de orina y la incontinencia. Las infecciones del tracto urinario (ITU) son más frecuentes en este grupo de pacientes, y así se refleja en nuestra casuística. Esta predisposición se debe a dos motivos: la enfermedad de base que sufren y la predisposición que supone el embarazo para infecciones de este tracto. Pero no sólo eso, a consecuencia del aumento de infecciones del tracto urinario, sobre todo de las crónicas, el riesgo de parto pretérmino aumenta. En general, en las pacientes con lesiones medulares no parece que los partos de pretérminos sean más frecuentes (3), aunque es evidente que el riesgo de infecciones urinarias incrementado en este grupo podría ocasionarlo, como en nuestros casos que está ligeramente aumentada. Es necesario dar consejos higiénicos para prevenir las infecciones, y conviene detectar y tratar la bacteriuria asintomática con analítica, sedimento, urinocultivo y antibiograma de orina mensualmente. Deben administrarse las pautas más cortas de tratamiento antibiótico posibles, aunque siempre como mínimo 7 días, para evitar seleccionar bacterias y causar resistencia. De forma profiláctica puede utilizarse la nitrofurantoína en el tercer trimestre para la bacteriuria crónica, si no se ha utilizado previamente en el embarazo. Hasta el momento no hay acuerdo en cuanto al manejo de las infecciones urinarias crónicas en estas gestantes.

Otro problema habitual para ellas, es el manejo de la incontinencia que es bastante complejo durante la gestación, ya que la mayoría de los tratamientos que se utilizan para este problema, no son seguros en el embarazo. Si los episodios de urgencia o incontinencia que sufren las pacientes aumentan durante el embarazo puede usarse bethanechol.

Debe evitarse el sondaje permanente, siendo preferibles los intermitentes, aunque es cierto que puede ser necesario para evitar el síndrome de hiperreflexia autónoma en el primer o tercer trimestre (1). No hay datos suficientes de seguridad en la gestación acerca de la estimulación de la raíz anterior, la neuromodulación sacra, el aumento del tamaño de la vejiga o la administración de toxina botulínica, aunque en este último hay algunos casos publicados que no han demostrado ningún problema con su uso en parapléjicas (5).

La función pulmonar de base de las pacientes con lesión espinal que desean gestar debería ser estudiada para identificar aquellas que pueden necesitar asistencia respiratoria en el parto $(3,4)$.
La capacidad de expansión pulmonar se ve limitada durante el embarazo por ganancia de peso, la curvatura espinal y la musculatura que afectan a la movilidad del diafragma, siendo este uno de los elementos más importantes en la respiración junto con los músculos cervicales, intercostales y abdominales. Además, las lesiones por encima de T10 tienen abolido incluso el reflejo tusígeno. Es recomendable hacer test de función respiratoria seriados durante la gestación, especialmente a aquellas mujeres que tienen afectada la región cervical. Si la capacidad vital alcanza valores por debajo de 13 $\mathrm{ml} / \mathrm{kg}$ a $15 \mathrm{ml} / \mathrm{kg}$ de peso pueden requerir incluso ventilación asistida. Otras medidas que pueden ayudar a las pacientes son la fisioterapia pulmonar, el uso de incentivadores pulmonares, el entrenamiento de los músculos accesorios respiratorios. Por otro lado, la posición en supino mejora la función respiratoria pero no es recomendable en estas gestantes porque puede provocar hipotensión y consecuentemente afectación fetal. Este conjunto de circunstancias hace que las infecciones del tracto respiratorio sean las más frecuentes en este grupo de pacientes y por supuesto, una causa considerable de morbimortalidad, llegando a desencadenar neumonia, atelectasia y edema pulmonar en algunas ocasiones, por lo que ante el mínimo síntoma o signo de infección, la paciente debe consultar y recibir el tratamiento adecuado para evitar un compromiso grave o una hospitalización (1). Pese a que la complicación más frecuente en estas gestaciones según consta en la literatura son las infecciones de vías respiratorias altas, en ningún caso en nuestra serie se produjeron.

La dilatación venosa como consecuencia de los niveles de progesterona, los cambios que se producen en la coagulación y la inmovilidad hacen que el riesgo de trombosis venosa profunda esté elevado en estas pacientes. Algunos estudios muestran una frecuencia del $8 \%$ en este grupo en comparación con el $0,1 \%$ en las gestantes sanas. Ciertos ejercicios pasivos de movimiento, elevación de las piernas, y el uso de medias de descompresión pueden disminuir el riesgo. No hay evidencia para recomendar tromboprofilaxis universal durante el embarazo y el puerperio en las pacientes con lesión medular, la evaluación y recomendaciones debe realizarse individualmente (en nuestra casuística solo lo requirieron tromboprofilaxis 2 pacientes) (3).

El dolor puede presentarse en cualquier gestante, pero debemos prestar especial atención a este tipo de pacientes porque muchas de ellas han sufrido, asociada a la lesión de la médula espinal, fracturas pélvicas, remodelación de las estructuras óseas, uso de tornillos o algún tipo de adhesivo en intervenciones previas. Generalmente, el paracetamol, seguro en el embarazo, no suele mejorar el dolor en estas pacientes. Una consulta con el neurólogo y el anestesista al inicio del embarazo puede 
ser útil en estas pacientes para manejar el dolor durante la gestación (1).

El manejo del dolor es importante, pero no es menos relevante la ausencia de este, la falta de sensibilidad de estas pacientes por debajo de la zona medular que tienen afectada conlleva que sean incapaces de percibir las contracciones y de identificar los primeros síntomas de inicio de parto. La capacidad de detectar el dolor producido por las contracciones depende de la altura a la que esté dañada la médula espinal. Mujeres con lesiones por debajo de T11 pueden percibirlo (3). Mujeres con lesiones entre T5 y T11 no pueden percibirlo, y deben ser monitorizadas para evitar un parto no controlado. Este tipo de pacientes puede sospechar que están alcanzando periodo activo de parto porque sufren espasmos flexores y espasticidad cuando alcanzan esta fase. Además el adiestramiento para realizar palpación uterina y detectar contracciones, el control con monitores en domicilio, la realización de tactos vaginales semanales a partir de cierta edad gestacional o el ingreso cerca de la fecha de término de la gestación pueden evitar esa situación. La percepción de un modo u otro de las contracciones en aquellas mujeres con lesiones por debajo de T5 hacen posibles los pujos y por tanto el parto vaginal si las condiciones son favorables y no lo impide ninguna otra condición obstétrica. En lesiones sobre T4 o por encima, la vía del parto será cesárea en todos los casos, porque no perciben las contracciones ni tienen capacidad de realizar pujos (3). En este grupo de pacientes la incidencia de cesáreas está muy aumentada, llegando al $58,82 \%$ en nuestra serie.

La complicación más grave de la gestante con lesión espinal es la hiperreflexia autónoma que se produce hasta en el $90 \%$ de las pacientes con lesión por encima de T6, y el $50 \%$ de estas incrementan la frecuencia de estos episodios en el embarazo (1), en lesiones medulares por debajo de esa región también se producen aunque no con tanta frecuencia. Se desencadena por irritación o distensión del cérvix, vagina, vejiga o intestino que produce una hiperactividad simpática por debajo del nivel de la lesión a consecuencia de que esa porción de la médula está desconectada del control hipotalámico. La clínica consiste en hipertensión maligna (no cursa con proteinuria a diferencia de la preeclampsia y es importante diferenciarlas porque habitualmente la tensión arterial se normaliza cuando cede el estímulo desencadenante), pérdida de la consciencia, cefalea occipital severa, midriasis, espasmos en los músculos de las extremida- des inferiores, congestión nasal, eritema facial, sudoración, piloerección, bradicardia o taquicardia y arritmias. Puede producirse también vasoconstricción úteroplacentaria conduciendo a hipoxia fetal. Es imprescindible la monitorización de la frecuencia cardiaca y la tensión arterial de las pacientes porque accidentes cerebrovasculares, hemorragia interventricular e infarto de miocardio pueden producirse. Este síndrome puede prevenirse mediante la administración de analgesia epidural hasta T10, incluso si la paciente no percibe dolor y mantenerla hasta 24 horas posparto. Por ese motivo, es necesario garantizar a la paciente una consulta con el equipo de anestesia previa al parto, para garantizar tenerlo todo previsto en el momento oportuno. Por otro lado, la simple colocación de una sonda Foley puede ser una medida profiláctica muy útil ya que asegura el vaciamiento continuo de la vejiga y evita la sobredistensión que puede desencadenar este evento (2). Si se produjese, es importante asegurarse de que la sonda está bien colocada y que drena adecuadamente evitando la sobredistensión de la vejiga, además, si la paciente no hubiese recibido anestesia epidural aún, puede ser útil colocarla en posición de sedestación, retirar las prendas ajustadas alrededor del cuello, evacuar el recto y administrar nitroglicerina en parche o en pasta. Si fuese necesario puede administrarse medicación antihipertensiva (nunca inhibidores del eje renina angiotensina, ese tipo de tratamiento podría descompensarlas hemodinámicamente). En un caso publicado se observó beneficio al administrar sulfato de magnesio a una mujer con esta complicación, pero la experiencia es insuficiente. En el puerperio otro estímulo que puede desencadenar la hiperreflexia autónoma es la lactancia materna con lo cual es necesario un control estricto durante los primeros días posparto y posteriormente, pero el riesgo no supera el beneficio que implica la lactancia materna de modo que se recomienda también en parapléjicas (1). En nuestra serie no hubo pacientes con hiperreflexia autónoma.

Habitualmente, los resultados obstétricos y perinatales obtenidos son muy positivos (en nuestra serie 18 recién nacidos sanos), lo que alienta a las pacientes y las anima a ser madres, esto hace que el número de gestantes en estas condiciones esté aumentando y son más frecuentes que hace 10-15 años.

Dada la bibliografía revisada y la casuística manejada en nuestro centro proponemos directrices para el manejo de estas pacientes, que se presentan en la Tabla II. 


\title{
Tabla II
}

RECOMENDACIONES DURANTE EL EMBARAZO, PARTOY PUERPERIO EN EMBARAZADAS PARAPLÉJICAS

\author{
Preconcepcional - Optimizar estatus físico y mental. \\ - Estudio genético si así lo determinan otros antecedentes personales o familiares. \\ - Estudio de la función pulmonar. \\ - Valoración y modificación si precisa de los tratamientos habituales. \\ - Ácido fólico 4 g/día si tiene tratamiento con fármacos antiepilépticos.
}

- Estudios de función pulmonar seriados: Fisioterapia pulmonar, uso de incentivadores pulmonares, entrenamiento de músculos accesorios respiratorios. Si la función pulmonar es $<13-15 \mathrm{ml} / \mathrm{kg}$ de peso puede requerir incluso ventilación asistida. Debe instruirse a la paciente a consultar ante cualquier indicio de infección respiratoria para recibir tratamiento adecuado y precoz.

- Riesgo de trombosis venosa profunda: Ejercicios pasivos de movimientos, elevación de miembros inferiores, uso de medias de compresión. Valorar recomendación de profilaxis antitrombótica con heparina de manera individualizada.

- Manejo del dolor: Consulta a neurología y anestesia al inicio del embarazo.

- Ansiedad y depresión: Terapia cognitivo conductual, acupuntura.

- Estreñimiento: Evacuación intestinal con supositorios, estimulación digital, ejercicios de evacuación, pautas dietéticas. Si es insuficiente añadir o aumentar dosis de ablandadores de heces suaves, agentes aumentadores de volumen y laxantes. Ocasionalmente requiere manejo multidisciplinar.

- Espasticidad y contracturas: Ejercicios de refuerzo de la musculatura en los miembros superiores pueden estar recomendados. Individualizar.

- Úlceras de decúbito: Se recomienda cambiar de postura frecuentemente dentro de la silla de ruedas, evitar la ropa con cremalleras, costuras o botones con áreas que hagan presión sobre la piel, hidratación abundante (al menos 8 vasos de agua al día), aumentar el consumo de calorías proteicas, utilizar humidificadores evitando la sequedad ambiental, limitar los baños y duchas con agua caliente, uso diario abundante de cremas hidratantes y lubricantes, colocar almohadillas de gel en zonas de presión, cambiar de postura los miembros elevándolos utilizando los brazos evitando arrastrarlos, crear un acolchado con almohadas entre los puntos de presión (rodillas y tobillos) durante el decúbito, masajear las zonas de presión, administrar $2000 \mathrm{UI} /$ día de vitamina D durante la gestación (recientemente recomendada, parece útil en mantener la integridad de la piel), utilizar una silla de ruedas de mayor tamaño que se adapte al nuevo peso y circunferencia de la mujer y asientos de gel o espuma que disminuyen la presión en la zona sacra y los muslos.

- ITU: Consejos higiénico dietéticos, detectar y tratar la bacteriuria asintomática (analítica, sedimento, urinocultivo y antibiograma de orina mensual), utilizar pautas antibióticas cortas pero nunca menores de 7-14 días, nitrofurantoína profiláctica durante el tercer trimestre.

- Incontinencia: Sondajes intermitentes en primer y segundo trimestre y permanente el tercer trimestre. Puede usarse bethanecol.

- Preparación al parto: Instruir en la palpación y detección de contracciones.

- Parto: Monitorización de la frecuencia cardiaca y la tensión arterial. Sonda Foley. Administrar anestesia epidural hasta T10 incluso si no percibe dolor y mantenerla hasta 24 horas posparto para evitar el síndrome de hiperreflexia autónoma.

- Lesiones sobre T5: Cesárea electiva.

- Lesiones entre T5 y T11: No perciben contracciones, ingreso cerca de la fecha de término para evitar un parto no programado. Es posible el parto vaginal.

- Lesiones por debajo de T11: Perciben contracciones. Es posible el parto vaginal.

- Síndrome de hiperreflexia autónoma: Administrar anestesia epidural, posición de sedestación, comprobar correcta colocación de sonda Foley, retirar prendas ajustadas alrededor del cuello, evacuar recto, administrar nitroglicerina. Si no fuese suficiente, puede administrarse tratamiento antihipertensivo (nunca IECAS). Valorar administración de sulfato de magnesio.

- Riesgo de trombosis venosa profunda: Ejercicios pasivos de movimientos, elevación de miembros inferiores, uso de medias de compresión. Valorar recomendación de profilaxis antitrombótica con heparina de manera individualizada. 


\section{CONCLUSIONES}

Las mujeres con lesiones medulares generalmente tienen buenos resultados obstétricos aunque sus gestaciones son de alto riesgo y requieren una atención multidisciplinar. La gestación debe ser planificada por la necesidad de una valoración respiratoria exhaustiva y de modificaciones en su tratamiento habitual. La complicación más grave es la hiperreflexia autonómica, pudiendo incluso llevar a la muerte de la madre y el feto. La necesidad de profilaxis antitrombótica debe valorarse individualmente. Una adecuada preparación física y mental para la gestación desde la consulta preconcepcional, junto con un abordaje multidisciplinar durante la gestación, el parto y el puerperio, hacen posible que gestantes parapléjicas puedan cumplir su deseo genésico, superando su discapacidad y alcanzando el gratificante objetivo de ser madre.

\section{REFERENCIAS}

1. Pardina $B$, Meje $T$, Villalonga A, Navarro M, García $\mathrm{CH}$, et al. Embarazo y parto en la mujer con lesión medular en fase crónica. Rev Esp Anestesiol Reanim 2011;48:93-6.

2. Barbara D. Challenges in the management of the pregnant woman with spinal cord injury. J Perinat Neonat Nurs 2013;27:225-31.

3. Sánchez-Alcalá EM. Gestantes con discapacidad: a propósito de un caso de mujer con lesión medular. Matronas Prof 2013;14:24-7.

4. American College of Obstetricians and Gynecologist. ACOG Committee Opinion: Number 275, September 2002. Obstetric management of patients with spinal cord injury. Obstet Gynecol 2002;100:625.

5. Pannek J, Bertschy S. Mission impossible? Urological management of patients with spinal cord injury during pregnancy: a systematic review. Spinal Cord 2011;49:1028-32. 